\title{
Letter to the Editor: Ultrastructural Changes in the Mitochondria in Disorders of Ornithine Metabolism
}

\author{
M. DARIA HAUST AND B. A. GORDON \\ Departments of Pathology [M. D. H.] and Biochemistry [B. A. G.], The Childrens' Psychiatric Research Institute, and \\ The University of Western Ontario, London, Ontario, Canada
}

In their recent paper, Shapiro et al. describe striking mitochondrial changes in the liver of a 17 -month-old female patient who was heterozygous for ornithine transcarbamylase (OTC) deficiency (7). Their ultrastructural studies of the hepatic mitochondria revealed "bud-like projections sausage-link appearance, elongation with short cristae, or the presence of parallel arrays of tubules," all of which the authors suggest have not been described previously in OTC deficiency. Their paper provides a valuable supplement to existing knowledge of the morphologic changes possible in the liver in OTC deficiency; however, the authors did not indicate that such changes have been previously reported in other metabolic diseases. Gatfield et al. (2) in a liver biopsy from a patient with the hyperornithinemia, hyperammonemia, and homocitrullinuria $(\mathrm{HHH})$ syndrome observed mitochondria with elongated forms and bizarre shapes containing parallel tubular structures with their long axis along the length of the mitochondria. In the mitochondria cut in cross-section, these tubules were observed to be peripherally disposed, separated from the inner mitochondrial membrane by an approximately 300 - $\AA$ thick sievelike membrane $(2,4)$. Small mitochondria with these same abnormal inclusions were observed to originate within the body of larger mitochondria. Mitochondria, elongated and segmented, appearing similar to the "sausage-link" deformity described by Shapiro et al. were observed by Arshinoff et al. (1) in the liver of two patients with hyperornithinemia and gyrate atrophy of the choroid and retina. Lesions similar to these latter were induced in rats by feeding a high ornithine diet (5).

The occurrence of abnormalities in mitochondrial ultrastructure similar to those described by Shapiro et al. (7) in other diseases prompts a search for metabolic derangements common to these diseases which could have played a pathogenetic role in these

Copyright (C) 1980 International Pediatric Research Foundation, Inc $0031-3998 / 80 / 1412-1411 \$ 02.00 \$ 0$ changes. Hyperornithinemia, which is probably a consequence of the defective transport of ornithine into the mitochondria in the HHH syndrome (3) and deficient ornithine keto acid transaminase in the hyperornithinemia and gyrate atrophy syndrome (6), is not generally observed in OTC deficiency. The efficacy of the urea cycle is compromised with impaired ammonia metabolism in both OTC deficiency and the HHH syndrome, but as Shapiro et al. suggest, these hapatic mitochondrial changes have not been encountered either in other primary urea cycle disorders or in other diseases associated with hyperammonemia such as Reye's syndrome.

\section{REFERENCES AND NOTES}

1. Arshinoff, S. A., McCulloch, J. C., Matuk, Y., Phillips, M. J., Gordon, B. A., and Marliss, E. B.: Amino-acid metabolism and liver ultrastructure in hyperornithinemia with gyrate atrophy of the choroid and retina. Metabolism, 28: 979 (1979).

2. Gatfield, P. D., Taller, E., Wolfe, D. M., and Haust, M. D.: Hyperornithinemia, hyperammonemia and homocitrullinuria associated with decreased carbamy phosphate synthetase I activity. Pediatr. Res., 9: 488 (1975).

3. Gordon, B. A. Gatield, P. D., and Wolfe, D. M. Studies on the metabolic defect in patients with hyperammonemia, hyperornithinemia and hemocitrullinuria. Clin. Res. (Abstract), 24: 668A (1976).

4. Haust, M. D., Gatfield, P. D., and Gordon, B. A.: Ultrastructure of hepatic mitochondria in a child with hyperornithinemia, hyperammonemia and homocitrullinuria. Hum. Pathol. (in press, February, 1981).

5. McCulloch, J. C., Arshinoff, S. A., Marliss, E. B., and Parker, J. A.: Hyperornithinemia and gyrate atrophy of the choroid and retina. Ophthalmology, 85: 918 (1978)

6. Sengers, R. C. A., Trijbels, J. M. F., Brussart, J. H., and Dreutman, A. F.: Gyrate atrophy of the choroid and retina and ornithine ketoacid aminotransferase deficiency. Pediatr. Res. (Abstract), 10: 894A (1976).

7. Shapiro, J. M., Schaffner, F., Tallon, H. H., and Gaull, G. E.: Mitochondrial abnormalities of liver in primary ornithine transcarbamylase deficiency. Pediatr. Res. 14: 735 (1980)

Printed in U.S.A 